Proceedings

\title{
A Novel Gyroscopic Actuation Concept for 2D MEMS Micromirrors ${ }^{+}$
}

\author{
Philip Kaupmann 1,*, Stefan Pinter ${ }^{1}$, Jochen Franz ${ }^{1}$, Reinhard Streiter ${ }^{2,3}$ and Thomas Otto ${ }^{2,3}$ \\ 1 Robert Bosch GmbH, Automotive Electronics, Reutlingen, Germany; \\ stefan.pinter@de.bosch.com (S.P.); jochen.franz@de.bosch.com (J.F.) \\ 2 Technical University of Chemnitz, Chemnitz, Germany; reinhard.streiter@zfm.tu-chemnitz.de (R.S.); \\ thomas.otto@enas.fraunhofer.de (T.O.) \\ 3 Fraunhofer Institute for Electronic Nano Systems (ENAS), Chemnitz, Germany \\ * Correspondence: Philip.kaupmann@de.bosch.com; Tel.: +49-7121-35-38176 \\ + Presented at the Eurosensors 2017 Conference, Paris, France, 3-6 September 2017.
}

Published: 9 August 2017

\begin{abstract}
In this paper we present a novel approach to achieve indirect quasistatic deflection of 2D MEMS scanning micromirrors by solely resonant excitation utilizing gyroscopic effects. Therefore the micromirror is set to oscillate in its mirror plane additionally to its primary resonant oscillation with a similar frequency. According to angular momentum conservation this leads to a quasistatic deflection along a third axis orthogonal to the former. To investigate the applicability to MEMS micromirrors we develop a reference MEMS design to be used for fully transient FEM simulation. To achieve consistent simulation results we further develop a closed loop control algorithm. We then perform simulations using this method to prove the viability of the proposed concept
\end{abstract}

Keywords: MOEMS; micromirror; indirect actuation; ansys; transient simulation

\section{Introduction}

MEMS scanning micro mirrors are an emerging technology with possible applications ranging from consumer pico projection [1,2] to 3D scanning [3], automotive HUD [4] as well as medical applications [5]. A large percentage of use cases requires or benefits from a 2D MEMS mirror concept that can be deflected about one resonant "fast" horizontal axis and a "slow" vertical static axis to be able to perform a regular multiline scan as depicted in Figure 1a. 2D MEMS micromirrors of this type were under development in several groups recently $[1,6,7]$.

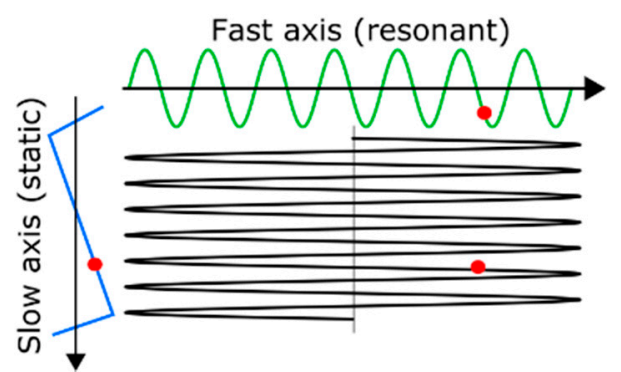

(a)

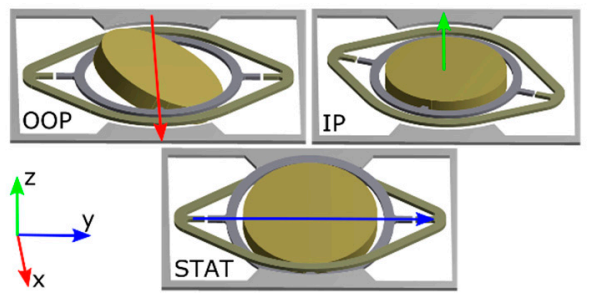

(b)

Figure 1. (a) Schematic representation of a 2D line scan projection; (b) Principle of the proposed actuation concept. The quasistatic (STAT) axis is indirectly driven by gyroscopic effects generated by actuation of the resonant OOP and IP axes. 
Designing a MEMS micro mirror to achieve a large static amplitude is challenging. Due to the absence of bearings one has to utilize torsional springs which are to be deflected by a static holding force obeying Hooke's Law. To achieve large deflection angles in a MEMS design this leads to either weak spring elements [8], possibly impairing vibration robustness, or the requirement of high forces. If these forces are generated by electrostatic drive concepts, voltages of up to several hundred volts are necessary [7]. An alternative to this is electromagnetic actuation. This approach whatsoever leads to a high current consumption and the need of one or several permanent magnets on chip or in package [1]. In our approach this dilemma is solved by using a merely resonant actuation in such a way that an indirect quasistatic deflection can be achieved exploiting gyroscopic effects as stated below.

\section{Novel Actuation Concept}

We suppose an abstract disc shaped mirror element with uniformly distributed mass and three rotational degrees of freedom, which are orthogonal to each other as depicted in Figure $1 \mathrm{~b}$. In a rotating reference frame, angular momentum conservation is described by

$$
\dot{I}+\omega \times I \omega=\tau, \omega=\left(\omega_{x}, \omega_{y}, \omega_{z}\right)^{T},
$$

with I: Angular inertia, $\omega$ : Angular velocity, $\tau$ : Torque [9]. If lumped torsion spring elements are added at all three degrees of freedom being subject to viscous damping, the equations of motion become

$$
\begin{aligned}
& I_{x} \ddot{\phi}_{x}(t)+\left(I_{z}-I_{y}\right) \dot{\phi}_{y}(t) \dot{\phi}_{z}(t)+D \dot{\phi}_{x}(t)+K_{x} \phi_{x}(t)=\tau_{x} \\
& I_{y} \ddot{\phi}_{y}(t)+\left(I_{z}-I_{x}\right) \dot{\phi}_{z}(t) \dot{\phi}_{x}(t)+D \dot{\phi}_{y}(t)+K_{y} \phi_{y}(t)=\tau_{y} \\
& I_{z} \ddot{\phi}_{z}(t)+\left(I_{y}-I_{x}\right) \dot{\phi}_{y}(t) \dot{\phi}_{x}(t)+D \dot{\phi}_{z}(t)+K_{z} \phi_{z}(t)=\tau_{z}
\end{aligned}
$$

where $\varphi$ : Deflection angle vector, $D$ : Damping coefficient, $K$ : Stiffness matrix. This set of differential equations consists of the harmonic oscillator equations with an additional gyroscopic coupling term G. If we now assume the body to be oscillating resonantly along two degrees of freedom ( $\mathrm{x}$ and $\mathrm{z}$ axis). Then $G$ will result in a torque $\tau_{G}=\left(I_{z}-I_{x}\right) \omega_{z}(t) \omega_{x}(t)$ in accordance to conservation of angular momentum. Since we assumed a resonant oscillation $\omega(t)=A \sin (\omega t)$, this torque can be converted using the trigonometric theorems:

$$
\tau_{G}=\frac{1}{2} A_{x} A_{z}\left(I_{z}-I_{x}\right)\left[\cos \left(\left(\omega_{z}-\omega_{x}\right) t\right)-\cos \left(\left(\omega_{z}+\omega_{x}\right) t\right)\right]
$$

If the angular velocities of $\mathrm{x}$ and $\mathrm{z}$ axis are equal, $\tau_{G}$ will have a constant bias as the first cosine term becomes equal to one. As the torque resulting from the second cosine is comparably high in frequency it will be mostly attenuated by viscous damping. The torque $\tau_{G}$ thus results in a quasistatic deflection of the micromirror, while actuated only resonantly. If the mirror element rotates with slightly different frequencies along the $\mathrm{x}$ and $\mathrm{z}$ axis respectively, the mirror will be deflected statically with the beat frequency $\left|\omega_{z}-\omega_{y}\right|$.

Specifically the micromirror will be designed to have a large $\mathrm{x}$ axis amplitude, thus acting as the horizontal mirror axis. The comparably large angular momentum of this oscillation is then disturbed by the oscillation along the $\mathrm{z}$ axis, resulting in a biased torque about the $\mathrm{y}$ axis, which over several ten to hundred periods leads to a quasistatic deflection. This is then used as the vertical mirror axis. As the $\mathrm{z}$ oscillation, parallel to the mirror surface normal, which in first approximation has no effect on the reflected beam pattern, its amplitude can be modulated to regulate the static axis amplitude respectively. With this approach it is possible to actuate the $2 \mathrm{D}$ micromirror by resonant drive concepts which are better suited for MEMS, while still deflecting it statically. This may lead to a significant reduction of power dissipation, as well as package dimensions of the device. 


\section{Modeling and Simulation}

To gain an understanding of the full dynamics of the postulated device, we developed a reference design of a gyroscopically actuated 2D MEMS micromirror as shown in Figure 2a using ANSYS Design Modeler. We decided to focus on a gimbal based design in which the degrees of freedom are separated by stiff cardan frames. The mirror element is attached to an inner cardan frame by two torsional springs representing the so called out of plane (OOP) rotational degree of freedom along the $\mathrm{x}$ axis. This inner part is able to rotate about the $\mathrm{y}$ axis via the static springs. These are attached to a second, heavier cardan frame that additionally acts as a counterbalance for the OOP rotation of the inner part. The rotational in plane (IP) degree of freedom is established by the four IP springs. These are dimensioned to allow the high strains expected because of the large displacement of the outer cardan frame even for relatively small IP angles. The outer ends of the IP springs are subject to zero displacement constraints. In order to enable a parameterization of the model with minimal change to the geometry we allowed a unique height for every design element within a reasonable range as feasible in a MEMS process.

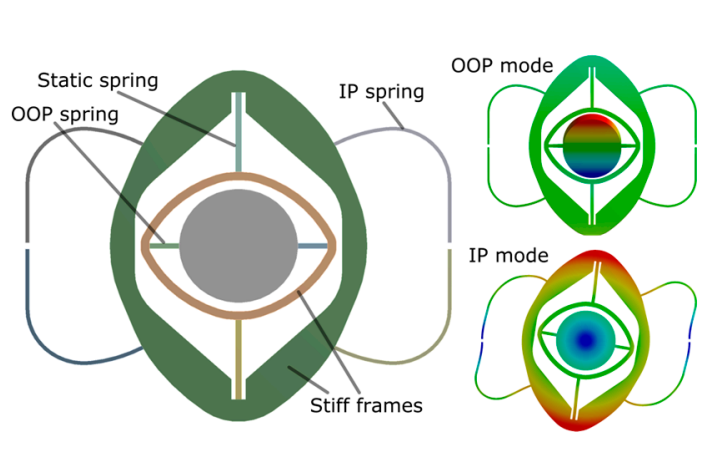

(a)

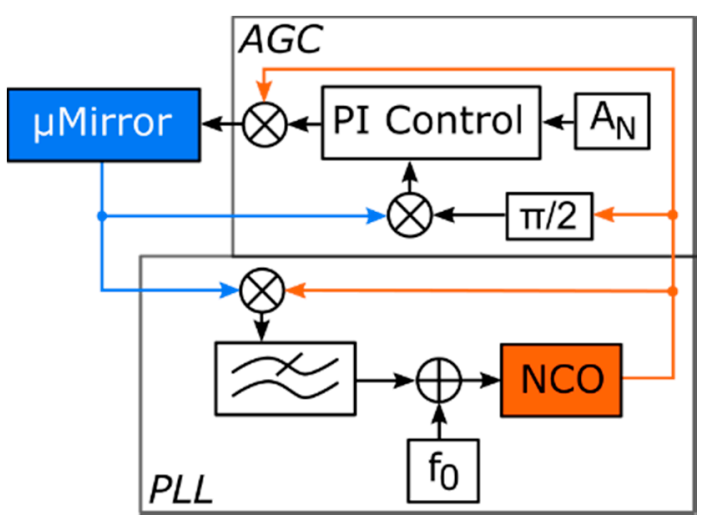

(b)

Figure 2. (a) CAD model of the developed reference design for a gyroscopically actuated 2D micromirror and shapes of the resonant modes of interest; (b) Schematic representation of the PLLAGC based control algorithm implemented in Ansys APDL.

We performed a modal analysis and adjusted the spring geometry parameters until achieving the desired mode shapes and frequencies. A central aspect besides setting the mode frequencies close to each other was to minimize the out of plane movement of the outer cardan frame as this possibly leads to an undesired coupling of OOP and IP modes. We therefore added more mass to the frame to keep the frame maximum $\mathrm{z}$ displacement less than $20 \%$ of the maximum mirror displacement. Additionally we had to design the geometry of the static spring in such a way that the resulting stiffness is higher than initially desired to keep its deformation resulting from the OOP and IP oscillations reasonably small. The resulting resonant drive modes of interest are shown in Figure 2a.

The reference design was then used to perform a full transient simulation using Ansys Mechanical Classic. In order to be able to set defined resonant amplitudes in full resonance a custom closed loop control was implemented in Ansys APDL as depicted in Figure 2b. In the phase locked loop (PLL) part a sinusoid signal with the initial frequency $f_{0}$ is generated by a numerically controlled oscillator (NCO). A phase detector unit provides the instantaneous phase relation between the generated and an output signal from the mirror model (i. e., the deflection angle). This is fed back in such a way that the NCO frequency is changed to establish a constant phase relation to the mirror model between the NCO signal used to actuate the mirror model and the model output signal [10]. The same demodulation technique is then utilized to extract the amplitude of the mirror output signal using an automatic gain control (AGC) consisting of an additional PI controller to regulate the nominal amplitude $A_{N}$. We realized two PLL-AGC entities running independently from each other, to control the OOP and IP axis separately. The transient simulation procedure was implemented as follows: In the first step only the OOP control loop is activated and the simulation is carried out until 
the OOP oscillation is in stationary state with the desired nominal amplitude of $A_{N, O O P}=8^{\circ}$ as depicted in Figure 5. We then defined a simulation restart point at this time to be able to repeat the following step with varying parameters. In the resumed simulation the IP axis control loop is then activated with a target amplitude of $A_{N, I P}=3^{\circ}$. Depending on the phase relation of OOP and IP axis an increasing deflection of the static axis is observable, that reaches a maximum amplitude of $A_{S T A T}=$ $2^{\circ}$ during the limited duration of the simulation (shown in Figure 3).

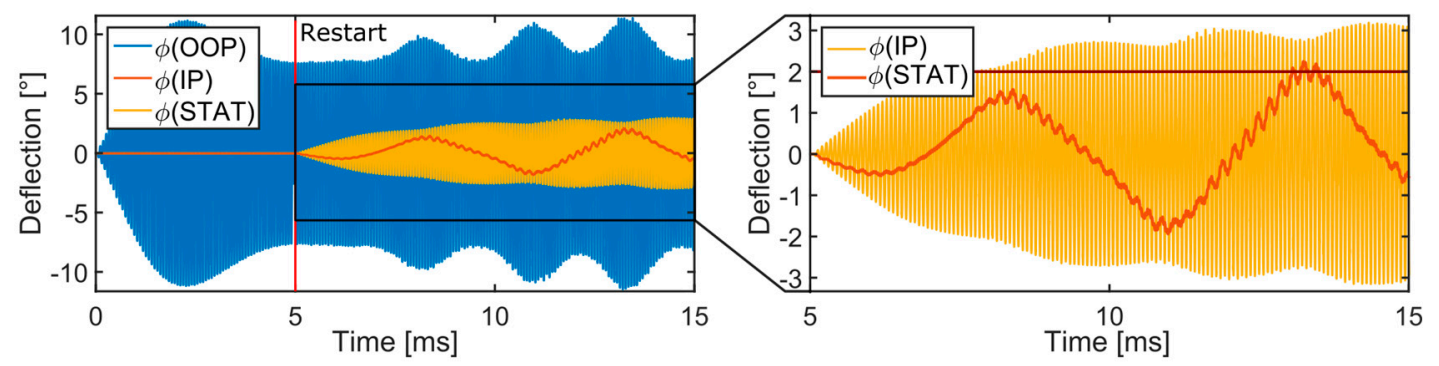

Figure 3. Result of a controlled transient simulation. At first a ramp up of the OOP axis is performed. At $t=5 \mathrm{~ms}$ the IP oscillation is activated, leading to an indirect quasistatic deflection of the STAT axis.

\section{Results and Conclusions}

We proposed a novel actuation concept for 2D MEMS micromirrors, allowing a potentially energy efficient indirect quasistatic deflection with merely resonant actuation by exploiting gyroscopic effects. To investigate the nonlinear dynamics of this type of micromirror we developed a reference design to carry out a fully transient FEM simulation study in Ansys Classic. To keep the system in resonance despite nonlinear frequency shifts and to control deflection amplitudes, we implemented a custom PLL-AGC based closed loop control algorithm in Ansys APDL. After finding practical solver parameters we ran transient simulations and achieved a quasistatic deflection of up to two degrees mechanical angle, proving the feasibility of the proposed actuation concept. Future work will include the design of a fully functional MEMS micromirror working with the proposed concept, and its manufacturing and characterization.

Conflicts of Interest: The authors declare no conflicts of interest

\section{References}

1. Niesten, M.; Masood, T.; Miller, J. Scanning laser beam displays based on 2D MEMS. Proc. SPIE 2010, 27723, doi:10.1117/12.851412.

2. Davis, W.O.; Sprague, R.; Miller, J. MEMS-Based Pico Projector Display. In Proceedings of the 2008 IEEE/LEOS International Conference on Optical MEMS and Nanophotonics, Freiburg, Germany, 11-14 August 2008.

3. Sandner, T.; Grasshoff, T.; Schenk, H. Quasi-static microscanner with linearized scanning for an adaptive 3D-laser camera. In Proceedings of the 2013 International Conference on Optical MEMS and Nanophotonics (OMN), Kanazawa, Japan, 18-22 August 2013.

4. Ben-Mrad, R.; Pasiliao, D. A MEMS micromirror based head-up display system. In Proceedings of the 2015 Symposium on Integration and Packaging of Mems/Moems (Dtip), Montpellier, France, 27-30 April 2015.

5. Qiu, Z. MEMS-based Medical Endomicroscopes. In Proceedings of the 2013 International Conference on IEEE Journal of Selected Topics in Quantum Electroncis, July-Augest 2015; pp. 376-391.

6. Ji, C.-H.; Ahn, S.-H.; Bu, J.-U. Dual-Axis Electromagnetic Scanning Micromirror Using Radial Magnetic Field. In Proceedings of the 19th IEEE International Conference on Micro Electro Mechanical Systems, Istanbul, Turkey, 22-26 January 2006.

7. Schock, W.; Mehner, J.; Fritz, J. FEM based modeling and optimization of a 2D micro mirror. In Proceedings of the EuroSimE, Linz, Austria, 18-20 April 2011. 
8. Tsai, Y.; Lee, J.; Gessner, T. Metallic glass micro-mirror integrated with PZT actuation for low resonant frequency and large exciting angle. In Proceedings of the 17th International Conference onSolid-State Sensors Actuators and Microsystems (TRANSDUCERS \& EUROSENSORS XXVII), Barcelona, Spain, 1620 June 2013.

9. Wittenburg, J. Dynamics of Multibody Systems; Springer: Berlin, Germany, 2008; pp. 45-46.

10. Proakis, J.G. Communication Systems Engineering; Prentice Hall: Bergen, NJ, USA, 2001; p. 113.

(C) 2017 by the authors. Licensee MDPI, Basel, Switzerland. This article is an open access article distributed under the terms and conditions of the Creative Commons Attribution (CC BY) license (http://creativecommons.org/licenses/by/4.0/). 